Revista Brasileira de Agricultura Irrigada v.11, nº.5, p. 1662 - 1669, 2017

ISSN 1982-7679 (On-line)

Fortaleza, CE, INOVAGRI - http://www.inovagri.org.br

DOI: 10.7127/rbai.v11n500745

Protocolo 745.17 - 23/05/2017 Aprovado em 31/08/2017

\title{
CALIBRAÇÃO DO SENSOR DE CAPACITÂNCIA DIVINER 2000 UTILIZANDO TUBOS DE ACESSO PADRÃO E ALTERNATIVO
}

Ronaldo Souza Resende ${ }^{1}$, Wagner Roberto Milet Batista ${ }^{2}$, Julio Roberto Araujo de Amorim ${ }^{3}$

\section{RESUMO}

Os equipamentos FDR utilizam tubos de acesso que não são similaridade no mercado nacional, resultando em elevado custo e tempo de aquisição para sua importação. O presente estudo teve como objetivo calibrar um sensor de capacitância Diviner $2000^{\circledR}$ para as condições de um solo Argissolo amarelo, com a utilização de tubo de acesso padrão do equipamento e de eletroduto de PVC rígido, alternativo ao tubo padrão. Foram abertas três trincheiras, correspondentes a um estado de umidade do solo - seco, intermediário e úmido. Para cada condição de umidade, instalaram-se quatro tubos de acesso: dois tubos adquiridos do fabricante do equipamento e dois do tipo eletroduto. A umidade medida com o eletroduto apresentou valores de RMSE superiores aos obtidos com o tubo original do equipamento. Os resultados evidenciaram, no entanto, a viabilidade da utilização do eletroduto, em substituição ao tubo padrão, desde que se realize a calibração local.

Palavras-chave: umidade do solo, sensor de capacitância, reflectometria no domínio da frequência.

\section{CALIBRATION OF DIVINER 2000 CAPACITANCE SENSOR USING STANDARD AND ALTERNATIVE ACCESS TUBES}

\begin{abstract}
FDR devices use access tubes that have no similar products available on the Brazilian market, resulting in the need for their importation with high cost and time of acquisition. Therefore, this study aimed to calibrate a capacitance sensor for a Cohesive Yellow Argisol conditions with the use of the standard access tube of the Diviner $2000^{\circledR}$ and with a rigid PVC conduit, as an alternative to the standard tube. Three trenches were opened in the soil, corresponding to a state of soil moisture, i.e., dry, intermediate and moist. For each soil moisture condition, four access tubes were installed, being two tubes acquired from the Diviner $2000^{\circledR}$ manufacturer and two others, of the conduit type, commercially available. The moisture content measured with the conduit tube showed square root values of mean square error (RMSE) higher than those
\end{abstract}

\footnotetext{
${ }^{1}$ Doutor, Pesquisador da Embrapa, Aracaju, Sergipe. Email: ronaldo.resende@embrapa.br.

${ }^{2}$ Mestre, Agrocana Com. e Representações Ltda., Maceió, Alagoas. Email: wagner@agrocana-ne.com.br.

${ }^{3}$ Mestre, Pesquisador da Embrapa, Aracaju, Sergipe. Email: julio.amorim@embrapa.br.
} 
obtained with the original tube from the device manufacturer. Without local calibration, the two types of tube tested resulted in overestimation of moisture. However, the results evidenced the feasibility of using the evaluated conduit tube instead of the standard one, since the field calibration is performed.

Keywords: soil water content, capacitance sensor, frequency domain reflectometry.

\section{INTRODUÇÃO}

Um correto manejo da água na agricultura irrigada pressupõe o monitoramento da umidade do solo com vistas a fornecer água às plantas no momento e na quantidade adequada, evitando o déficit e o excesso hídrico. No campo da pesquisa, ocorreu nos últimos anos uma clara tendência de redução do uso da sonda de nêutrons, dado os inconvenientes da utilização de material radioativo para o seu funcionamento. Assim, ganharam espaço os equipamentos para medição da umidade do solo baseados nos princípios da reflectometria no domínio do tempo e no domínio da frequência (conhecidos respectivamente por suas siglas em inglês TDR e FDR), os quais exploram a relação entre o conteúdo de água no solo e a constante dielétrica do solo (Evett et al., 2006).

Essa técnica se baseia no fato de que a água apresenta um valor de constante dielétrica $(\xi)$ bem maior $(\sim 80)$ do que dos principais constituintes da matriz do solo ( 4) e do ar $(\sim 1)$ (Gaskin \& Miller, 1996). Andrade et al. (2008) apontam uma série de vantagens e desvantagens associadas à técnica da determinação da umidade do solo por meio do princípio da FDR, sendo que uma das desvantagens se refere à necessidade de calibração local. Em vários estudos, efetuaramse calibrações locais de equipamentos FDR para diferentes tipos de solo (Silva et al., 2007; Lima et al., 2012; Silva et al., 2012; Silva Junior et al., 2013).

Em função da baixo valor da constante dielétrica do ar em relação à da água, além do processo de instalação e manutenção do tubo de acesso, no que se refere ao contato com o solo imediatamente ao seu redor (George, 1999), o seu diâmetro interno tem importância na acurácia da medição em função do grau de interferência da camada de ar, na interface entre a superfície do sensor e a parede interna do tubo, conforme preconizado por Andrade et al. (2008).

Um aspecto relacionado aos equipamentos FDR que utilizam tubos de acesso de PVC, como é o caso do modelo Diviner $2000^{\circledR}$ (fabricado pela Sentek Pty Ltd, Autrália), é o fato de que normalmente não são encontrados tubos similares no mercado nacional, principalmente em função do seu diâmetro interno e espessura de parede. Isso resulta na necessidade de importação de tubos de acesso fornecidos pelo fabricante do equipamento, com custo e tempo de aquisição elevados, limitando assim o aproveitamento do sistema adquirido.

Este estudo teve como objetivo efetuar a calibração de um sensor de capacitância para as condições de um Argissolo amarelo coeso dos Tabuleiros Costeiros do Estado de Sergipe, bem como avaliar a utilização de um eletroduto de PVC rígido como alternativa ao tubo de acesso padrão do Diviner $2000^{\circledR}$.

\section{MATERIAL E MÉTODOS}

O experimento foi conduzido no Campo Experimental de Umbaúba, pertencente à Embrapa Tabuleiros Costeiros e localizado no Município de Umbaúba, SE, cujas coordenadas geográficas são $11^{\circ} 22^{\prime} 37^{\prime}$ ' de latitude Sul e $37^{\circ}$ 40' 26', de longitude Oeste, com altitude média de $109 \mathrm{~m}$.

Segundo a classificação de Köppen, o clima da região é caracterizado como “As”, ou seja, tropical chuvoso com verão seco. Com temperatura média de $24,1^{\circ} \mathrm{C}$, umidade relativa de $81,4 \%$, e precipitação pluvial média de $1.317,9 \mathrm{~mm}$, conforme dados fornecidos pela Estação Agrometereorológica do local. O solo é classificado como Argissolo Acinzentado Eutrófico, com fragipã, textura média a argilosa e relevo plano. Conforme Cintra (2017), os 
Argissolos são predominantes na unidade de paisagem dos Tabuleiros Costeiros do Nordeste do Brasil.

Foram abertas três trincheiras com dimensões de $2 \mathrm{~m} \mathrm{x} 1 \mathrm{~m}$ x $1 \mathrm{~m}$ (comprimento $\mathrm{x}$ largura $\mathrm{x}$ profundidade), distanciadas $3 \mathrm{~m}$ entre si. Cada trincheira correspondeu a um estado de umidade do solo, sendo denominados: seco, intermediário e úmido. Para obtenção da condição de solo intermediário e úmido, foram instalados anéis de aço no solo, sendo aplicado no seu interior volume de água suficiente para umedecimento completo do perfil.

Seguindo as recomendações do manual do fabricante da sonda (Sentek, 2000), foram instalados quatro tubos de acesso para cada condição de umidade do solo, sendo dois tubos adquiridos do fabricante do equipamento e dois do tipo eletroduto (Figura 1). O eletroduto de PVC rígido, avaliado como alternativa ao tubopadrão, é comercializado em barras de $3 \mathrm{~m}$, na cor preta, com tratamento antichama recomendado para instalações elétricas embutidas, normatizado com NBR 15465. Uma vez que o diâmetro externo do eletroduto é maior do que a do tubo-padrão, não foi possível utilizar o kit de instalação que acompanha o equipamento. No entanto, não houve dificuldade para o seu correto alinhamento vertical. As características dos tubos avaliados são apresentadas na Tabela 1, com as dimensões representando a média de quatro leituras com paquímetro digital.

Para o perfil úmido, as leituras foram efetuadas no dia seguinte ao umedecimento do perfil do solo; para o perfil intermediário, cinco dias após o umedecimento. Como o experimento foi realizado na estação seca da região, as leituras para o perfil seco foram feitas no primeiro momento.

Para cada ponto, foram efetuadas duas leituras de frequência relativa (FR) da sonda Diviner $2000^{\circledR}$, totalizando quatro leituras por condição de umidade e para cada tubo de acesso avaliado, sendo utilizada a média aritmética delas para fins de calibração. A FR é obtida a partir da leitura de frequência efetuada pela sonda na camada considerada e relacionada com valores parametrizados no equipamento, conforme o modelo $\mathrm{FR}=(\mathrm{Fa}-\mathrm{Fs}) /(\mathrm{Fa}-\mathrm{Fw})$, em que $\mathrm{Fa}$ e $\mathrm{Fw}$ são a frequência lida com a sonda no interior do tubo de acesso suspenso no ar e mergulhado na água, respectivamente, e Fs é a frequência lida no solo.

As leituras de FR foram efetuadas em camadas de 0,1 $\mathrm{m}$ até a profundidade de $0,5 \mathrm{~m}$. Imediatamente após a leitura da umidade pela sonda, foram coletadas amostras de solo indeformadas nos pontos de cada tubo de acesso e nas mesmas camadas em que foram realizadas as medições de umidade para determinação da umidade volumétrica $\left(\Theta_{\mathrm{v}}\right)$, com o fim de correlacioná-las com as leituras da FR. Para a condição de solo seco, foram obtidas amostras deformadas, sendo determinada a umidade gravimétrica e, a partir da densidade do solo obtida nas demais amostras, calculada a umidade volumétrica.

Para verificação da precisão dos valores preditos pelo modelo default do equipamento e pelos modelos calibrados localmente em relação aos valores observados ( $\Theta_{v}$ gravimétrica), foram calculados a raiz quadrada da média dos quadrados dos erros (RMSE) e o índice refinado de Willmott $\left(\mathrm{d}_{\mathrm{r}}\right)$, adimensional (Willmott et al., 2012), o qual se constitui em uma reformulação do índice de Willmott (d), mas com maior amplitude, de -1 a 1 (Oliveira, 2016). Com o produto do índice $\mathrm{dr}$ pelo coeficiente de correlação de Pearson (r), obteve-se o índice de desempenho (Pi) dos modelos avaliados, conforme preconizado por Camargo \& Sentelhas (1997) e Alvares et al. (2013).

\section{RESULTADOS E DISCUSSÃO}

Os coeficientes da equação de calibração a e b dos modelos, obtidos para as diferentes profundidades, bem como a RMSE associada às estimativas de cada modelo, em relação ao método gravimétrico, são apresentados na Tabela 2, tanto para o tubo-padrão do equipamento como para o eletroduto. Embora alguns estudos apresentem as equações de ajuste da calibração considerando a frequência relativa como variável explanatória, optou-se aqui por apresentar os 
coeficientes das equações de ajuste no formato $\mathrm{FR}=\mathrm{a} \times \Theta_{\mathrm{v}}^{\mathrm{b}}$, de modo idêntico ao disponibilizado no manual do fabricante, e considerando, ainda, ser dessa forma que o equipamento é parametrizado.

Para o tubo de acesso padrão, os valores dos coeficientes dos modelos obtidos na calibração local foram próximos àqueles da calibração default do equipamento, exceto para as camadas mais profundas. Para todas as camadas, o erro associado à calibração local foi inferior à calibração default do equipamento, sendo a diferença maior nas camadas mais superficiais do solo, onde o nível de umidade era menor. Ao se considerar todo o perfil do solo, o valor da RMSE obtido com a calibração default do equipamento foi $130 \%$ maior do que o obtido com a calibração local.

Com base no manual do equipamento, a equação de calibração default é baseada em dados combinados de solos de textura arenosa, média e de solo orgânico (Sentek, 2000). O valor da RMSE de 0,015 obtido para a calibração local com o tubo padrão foi similar ao valor de 0,014 obtido por Silva et al. (2007) e inferior aos valores de 0,030 e 0,053 para as condições de solo seco ao ar e saturado, respectivamente, obtidos por Evett et al. (2006), todos utilizando o Diviner 2000.

A utilização do eletroduto com a aplicação do modelo default do equipamento resultou em elevado valor da RMSE para todas as profundidades do solo. Quando se considerou todo o perfil do solo, o valor da RMSE obtido com a calibração default do equipamento foi 7,2 vezes maior que o obtido com a calibração local. No entanto, os valores de RMSE obtidos para o eletroduto com a utilização da calibração local foram inferiores àqueles obtidos com o uso do tubo-padrão e a calibração default, bem como próximos aos observados para a calibração local associada ao tubo-padrão.

O índice de concordância refinado de Willmott (dr), o coeficiente de correlação de Pearson (r) e o índice de desempenho (Pi) para quantificação da aderência da estimativa da umidade obtida em relação ao método-padrão são apresentados na Tabela 3. Para o tubo-padrão com uso do modelo default, observa-se um valor de Pi de 0,469, classificado como "bom", conforme classificação encontrada em Alvares et al. (2013). O pior desempenho foi obtido pelo uso do eletrotubo combinado com o modelo default do equipamento, com valor de $\mathrm{Pi}$ de 0,251, considerado como "péssimo".

O uso dos modelos obtidos localmente refletiu em melhoria da concordância dos valores de umidade estimados em relação ao métodopadrão. Ao se considerar todo o perfil do solo estudado, o uso da calibração local resultou em desempenho semelhante para os dois tubos avaliados, com valores de Pi igual a 0,678 para o tubo-padrão e a 0,721 para o eletrotubo, sendo ambos classificados como "muito bom". O equipamento Diviner 2000 permite ao usuário a especificação de modelos calibrados específicos para diferentes camadas de solo. O uso desse procedimento resultou em elevação dos valores de $\mathrm{dr}$ e $\mathrm{r}$ para os dois tubos (Tabela 3), com consequente melhoria do desempenho da medição e enquadramento dos valores de $\mathrm{Pi}$ como "ótimo", também para os dois tubos avaliados.

Os valores de umidade do solo obtidos pelo método gravimétrico foram plotados com os valores estimados pelo modelo default do equipamento e pelo calibrado localmente, para o tubo de acesso padrão e para o tubo eletroduto (Figura 2). Considerando-se os modelos obtidos para todo o perfil do solo, observa-se que, em termos médios, a utilização da calibração default forneceu valores de umidade do solo superestimados para ambos os tipos de tubo, sendo essa superestimativa de 1,2 vezes para o tubo-padrão e de 1,8 vezes para o eletroduto, aproximadamente. Já a utilização da calibração local resultou em uma melhor aderência dos valores preditos em relação à reta 1:1 para os dois tubos avaliados. Nesse caso, observava-se que os valores preditos pelos modelos subestimaram a umidade do solo também para os dois tubos, na ordem de $3 \%$ para o tubo-padrão e de $4 \%$ para o eletroduto, considerando-se a camada de $0-50$ m.

As curvas de calibração obtidas com o uso do tubo padrão e do eletroduto são apresentadas na Figura 3. O eletroduto resultou em medidas mais elevadas de frequência relativa para todo o 
intervalo de umidade do solo, sendo a diferença entre ambas significativa pelo teste $t(\mathrm{p}<0,01)$. No entanto ambas apresentaram acurácia similar na estimativa da umidade do solo, com valores de RMSE de $0,0153 \mathrm{~m}^{3} \mathrm{~m}^{-3}$ para o tubo padrão e de $0,158 \mathrm{~m}^{3} \mathrm{~m}^{-3}$ para o eletroduto. Observa-se que a diferença entre os dois ajustes se mantêm aproximadamente constante em todo o intervalo de umidade, ou seja, nas condições de solo seco e úmido.

O contato do tubo de acesso com o solo influencia a acurácia e precisão da medida da umidade do solo com uso de sondas de capacitância (EVETT et al., 2006), bem como contato da sonda medidora com a parede interna do tubo. A diferença de diâmetro interno entre o tubo padrão e o eletroduto aqui avaliado é de 1,09 $\mathrm{mm}$ (Tabela 2), significando $0,54 \mathrm{~mm}$ a mais de camada de ar na interface eletroduto-sonda. Dois dos principais fabricantes nacionais de tubos PVC ofertam linha para água (PBA - Classe 20) o qual possui diâmetro interno próximo ao do tubo padrão, com 51,4 mm. Embora tenham maior espessura de parede, ambos com 4,3 mm, poderiam futuramente também ser testados.

\section{CONCLUSÕES}

1. Para as condições prevalecentes no estudo, a calibração local do equipamento tornase necessária quando o interesse é a determinação do valor absoluto da umidade do solo, com o fim de se obter maior precisão.

2. A utilização de calibração específica para diferentes camadas do solo melhora de forma significativa o desempenho da medida da umidade do solo.

3. O uso do eletroduto como substitutivo do tubo de acesso padrão do equipamento somente pode ser efetuado com a calibração local.

4. A calibração local torna viável a utilização do eletroduto em substituição ao tubopadrão do equipamento.

\section{REFERÊNCIAS BIBLIOGRÁFICAS}

ALVARES, C.A.; STAPE, J.L.; SENTELHAS, P.C.; DE MORAES GONÇALVES, J.L.
Modeling monthly mean air temperature for Brazil. Theoretical and Apllied Climatology, v. 113, n. 3-4, p. 407-427, 2013.

ANDRADE, C. de L.T. de; BORGES JUNIOR, J.C.F.; COUTO, L. Características físicohídricas e dinâmica da água no solo. In: ALBUQUERQUE, P. E. P. de; DURÃES, F. O. M. (Ed.). Uso e manejo de irrigação. Brasília, DF: Embrapa Informação Tecnológica; Sete Lagoas: Embrapa Milho e Sorgo, 2008. cap. 2, p. 71-149.

CAMARGO, A.P.; SENTELHAS, P.C. Avaliação de diferentes métodos de estimativa da evapotranspiração potencial no Estado de São Paulo, Brasil. Revista Brasileira de Agrometeorologia, v. 5, n. 1, p. 89-97, 1997.

CINTRA, F.L.D. Solos dos Tabuleiros Costeiros. In: Árvore do conhecimento: coco. Disponível em: <http://www.agencia.cnptia.embrapa.br/gestor/c oco/arvore/ CONT000gm3htqb402 wx5ok0f7mv20chka6ca.html>. Acesso em: 15 jun. 2017.

EVETT, S. R.; TOLK, J. A.; HOWELL, T.A. Soil profile water content determination: sensor accuracy, axial response, calibration, temperature dependence and prcision. Vadose Zone Journal, n.5, p.894-907, 2006.

GASKIN, G.J; MILLER, J.D. Measurement of Soil Water Content Using a Simplified Impedance Measuring Technique. Journal of Agricultural Engineering Research, v. 63, n.2, p.153 - 159, 1996.

GEORGE, B.H. Comparison of techniques for measuring the water content of soil and other porous media. 1999. 192p. Dissertação (Mestrado). University of Sidney, New South Wales, 1999.

LIMA, J.E.F.W; SILVA, E.M. da; KOIDE, S.; SANTOS, R.M. dos. Avaliação do desempenho 
de sonda de capacitância no monitoramento da umidade de Latossolos do Cerrado em condições de campo. Revista Brasileira de Recursos Hídricos, v. 17, n. 1, p. 23-32, janeiro/março, 2012.

OLIVEIRA, E.A. de. Métodos para análise de concordância: estudo de simulação e aplicação a dados de evapotranspiração. 2016. 177 p. Tese (Doutorado) - Escola Superior de Agricultura “Luiz de Queiroz”, Universidade de São Paulo, Piracicaba, 2016.

SENTEK. Diviner 2000: user guide version 1.21. Stepney: Sentek Pty Ltd., 2000.

SILVA JUNIOR, J.J.; COLOMBO, A.; SCLALCO, M.S.; SILVA, B.M.; LIMA, P.T. Calibração de sondas de capacitância para determinação de umidade em Latossolo Vermelho distróférrico. Irriga, v. 18, n. 4, p. 743755, outubro/dezembro, 2013.
SILVA, B.M.; OLIVEIRA, G.C. de; SERAFIM, M.E.; SILVA JÚNIOR, J.J. da; COLOMBO, A.; LIMA, J.M. de. Acurácia e calibração de sonda de capacitância em Latossolo Vermelho cultivado com cafeeiro. Pesquisa Agropecuária Brasileira, v. 47, n. 2, p. 277-286, fev. 2012

SILVA, C.R. da; ANDRADE JUNIOR, A.S.de; ALVES JÚNIOR, J.; SOUZA, A.B. de; BRITO MELO, F. de; COELHO, M.A. Calibration of a capacitance probe in a paleudult. Scientia Agricola, v. 64, n. 6, p. 636-640, novembro/dezembro, 2007.

WILLMOTT, C.J.; ROBESONB, S.M.; MATSUURA, K. Short comunication: a refined index for model performance. Intl. Journal of Climate, Malden, v.32, p.2088-2094, 2012.

WIILMOTT, C.J.; ROBESONB， S.M.; MATSUURA, K. Short comunication: a refined index for model performance. Intl. Journal of Climate, Malden, v.32, p.2088-2094, 2012.
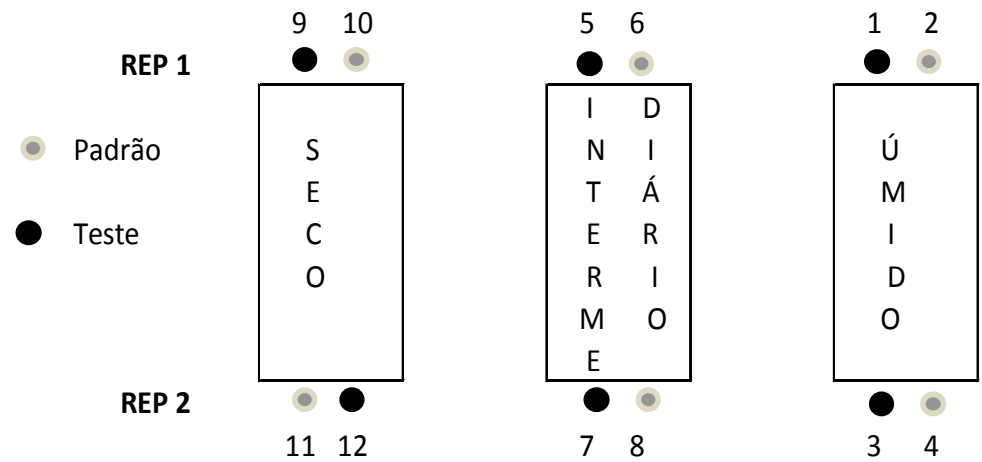

Figura 1. Esquema de instalação dos tubos de acesso para cada condição de umidade do solo e dos anéis de infiltração para umedecimento artificial do perfil do solo.

Tabela 1. Características físicas dos tubos de acesso utilizados na calibração do sensor de capacitância Diviner $2000^{\circledR}$.

\begin{tabular}{|c|c|c|}
\hline Característica & Tubo Diviner & Eletroduto \\
\hline Diâmetro externo - De (mm) $)^{1}$ & 56,51 & 59,14 \\
\hline Diâmetro interno - Di (mm) & 51,19 & 52,28 \\
\hline Espessura de parede $(\mathrm{mm})^{1}$ & 2,66 & 3,43 \\
\hline Cor & Branca & Preta \\
\hline
\end{tabular}

${ }^{1}$ Média de 10 medições com paquímetro digital 
Tabela 2. Coeficientes dos modelos calibrados localmente, amplitude de umidade do solo e valores de RMSE, considerando-se a calibração local e a default do equipamento.

\begin{tabular}{|c|c|c|c|c|c|c|c|c|}
\hline $\begin{array}{c}\text { Camada } \\
\text { (m) }\end{array}$ & $\mathbf{a}^{1}$ & $\mathbf{b}^{1}$ & $\overline{\mathbf{R}^{2}}$ & $\mathbf{N}^{1}$ & \multicolumn{2}{|c|}{$\begin{array}{c}\text { Amplitude } \Theta_{\mathrm{v}} \\
\left(\mathrm{m}^{3} \mathrm{~m}^{-3}\right)\end{array}$} & $\begin{array}{l}\text { RMSE }^{1} \\
\text { local }\end{array}$ & RMSE default \\
\hline Default & 0,2746 & 0,3314 & 0,99 & - & & & - & - \\
\hline \multicolumn{9}{|c|}{ Tubo-padrão } \\
\hline $0-0,1$ & 0,2874 & 0,3496 & 0,93 & 6 & 0,044 & 0,127 & 0,0087 & 0,0324 \\
\hline $0,1-0,2$ & 0,2645 & 0,3779 & 0,92 & 6 & 0,056 & 0,136 & 0,0076 & 0,0304 \\
\hline $0,2-0,3$ & 0,2719 & 0,3587 & 0,57 & 6 & 0,080 & 0,150 & 0,0164 & 0,0405 \\
\hline $0,3-0,4$ & 0,2817 & 0,3428 & 0,72 & 6 & 0,107 & 0,185 & 0,0157 & 0,0391 \\
\hline $0,4-0,5$ & 0,4877 & 0,1331 & 0,10 & 6 & 0,128 & 0,190 & 0,0231 & 0,0364 \\
\hline $0-0,5$ & 0,3073 & 0,3083 & 0,81 & 30 & 0,044 & 0,190 & 0,0153 & 0,0359 \\
\hline \multicolumn{9}{|c|}{ Eletroduto } \\
\hline $0-0,1$ & 0,3929 & 0,2591 & 0,94 & 6 & 0,040 & 0,138 & 0,0133 & 0,0741 \\
\hline $0,1-0,2$ & 0,3960 & 0,2835 & 0,90 & 6 & 0,046 & 0,141 & 0,0112 & 0,1138 \\
\hline $0,2-0,3$ & 0,5222 & 0,1686 & 0,88 & 6 & 0,079 & 0,159 & 0,0106 & 0,1251 \\
\hline $0,3-0,4$ & 0,5229 & 0,1661 & 0,45 & 5 & 0,114 & 0,187 & 0,0204 & 0,1240 \\
\hline $0,4-0,5$ & 0,5545 & 0,1463 & 0,35 & 5 & 0,124 & 0,20 & 0,0220 & 0,1240 \\
\hline $0-0,5$ & 0,4136 & 0,2527 & 0,88 & 28 & 0,040 & 0,200 & 0,0158 & 0,1131 \\
\hline
\end{tabular}

${ }^{1}$ Os coeficientes $a$ e $b$ se aplicam à equação $\mathrm{FR}=\mathrm{a} \times \Theta_{\mathrm{v}}{ }^{\mathrm{b}}$, em que FR e $\Theta_{\mathrm{v}}$ são respectivamente a frequência relativa (adimensional) e a umidade volumétrica do solo (\%); N - número de amostras; RMSE - raiz quadrada da média dos quadrados dos erros.

Tabela 3. Relação entre os valores de umidade determinados pelo método gravimétrico e os preditos pelo modelo default do equipamento e pelos modelos calibrados localmente, para cada tubo de acesso avaliado.

\begin{tabular}{lccccccc}
\hline Índice/ & \multicolumn{3}{c}{ Tubo-Padrão $_{\text {Calibração }}$} & Default & Local1 $^{\mathbf{1}}$ & & \multicolumn{3}{c}{ Local2 $^{2}$} & & Default & Local1 $^{\mathbf{1}}$ & Local1 $^{\mathbf{2}}$ \\
\hline $\mathrm{d}_{\mathrm{r}}{ }^{3}$ & 0,542 & 0,781 & 0,822 & & $-0,278$ & 0,795 & 0,839 \\
$\mathrm{r}^{3}$ & $0,865^{* *}$ & $0,868^{* *}$ & $0,931^{* *}$ & & $0,905^{* *}$ & $0,907^{* *}$ & $0,942^{* *}$ \\
$\mathrm{P}_{\mathrm{i}}{ }^{3}$ & 0,469 & 0,678 & 0,765 & & $-0,251$ & 0,721 & 0,790 \\
\hline
\end{tabular}

${ }^{1}$ Local1 - utilização do modelo ajustado considerando todo o perfil do solo (0 - 0,5 m).

${ }^{2}$ Local2 - utilização do modelo ajustado considerando cada camada do solo (0,10 m).

${ }^{3} \mathrm{~d}_{\mathrm{r}}$ - índice de concordância refinado de Willmott; $\mathrm{r}$ - coeficiente de correlação de Pearson; Pi - índice de desempenho.

**Significativo, pelo teste $t$, ao nível de $99 \%$ de probabilidade. 


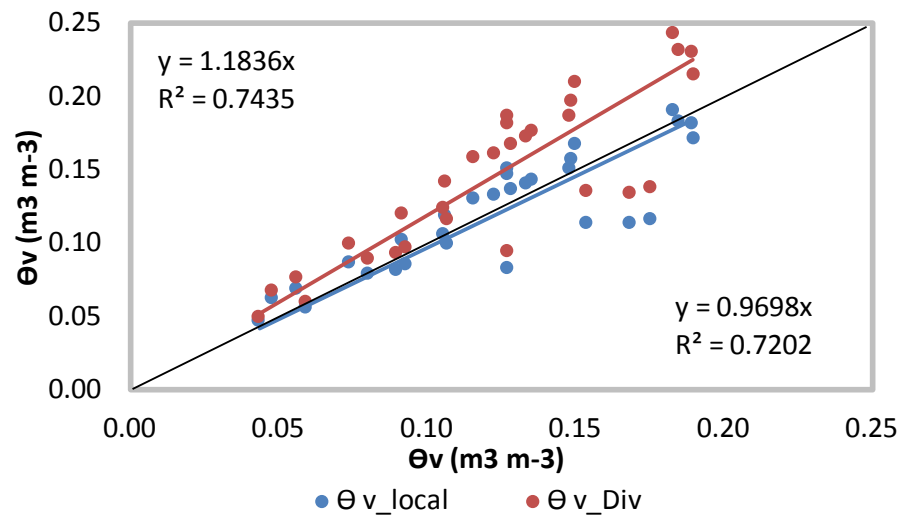

a

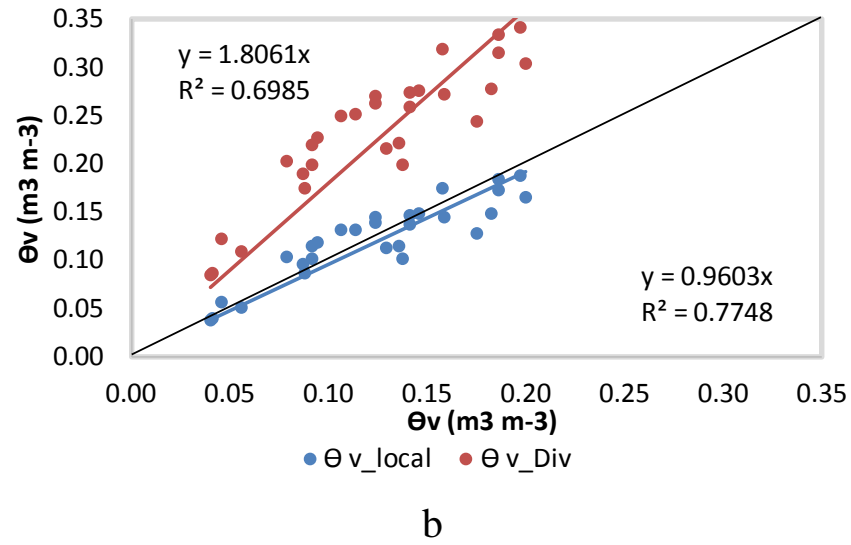

Figura 2. Relação entre a umidade do solo $\left(\theta_{\mathrm{v}}\right)$ medida pelo método gravimétrico e pelos tubos de acesso padrão (a) e eletroduto (b).

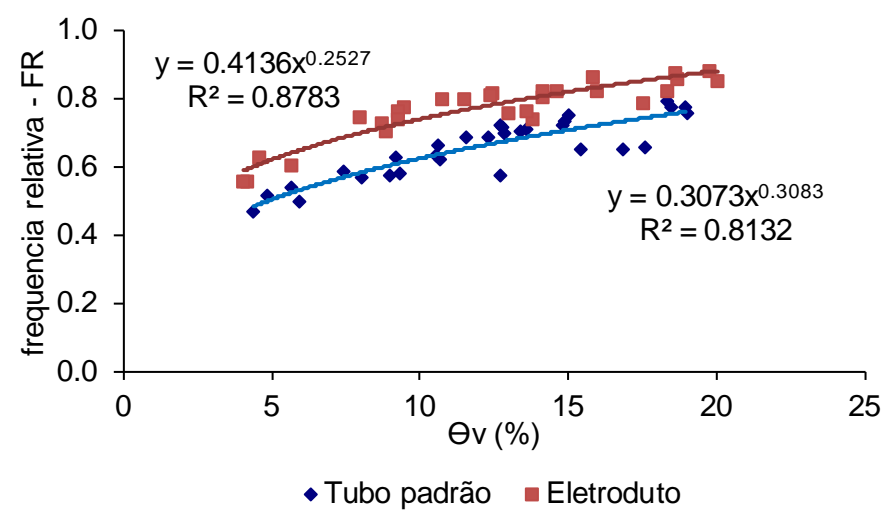

Figura 3. Curvas de calibração do Diviner 2000 em Argissolo amarelo, para os tubos de acesso padrão e eletroduto. 